\title{
The Dilemma of Myopericarditis Versus Acute Coronary Event
}

\author{
George Habib ${ }^{\mathrm{a}, \mathrm{d}}$, Mark Chernin ${ }^{\mathrm{b}}$, Jacob Goldstein ${ }^{\mathrm{c}}$
}

\begin{abstract}
Myopericarditis is an uncommon medical condition characterized by symptoms and signs of pericarditis with myocardial involvement. The main differential diagnosis is acute coronary event. Chest pain, electrocardiogtaphic changes, elevated cardiac enzymes and regional wall motion abnormality might turn the situation into a state of emergency. Here we describe a patient who presented with all of the above and positive family history of coronary artery disease. He was rushed to the catheterization unit, showing normal coronaries. Cardiac computerized tomography done later showed normal coronaries and pericardial enhancement typical of pericarditis with eventual diagnosis of myopericarditis due to streptococcal tonsillar infection. Patient was eventually treated with aspirin with full recovery of the left ventricular function.
\end{abstract}

Keywords: Myopericarditis; Acute coronary event; Streptococcal infection; Pericardial enhancement

\section{Introduction}

Myopericarditis is an uncommon medical condition characterized by symptoms and signs of pericarditis with myocardial involvement. It could be associated with infectious etiologies, mainly viral, like adenovirus [1], echovirus [2]

\footnotetext{
Manuscript accepted for publication June 6, 2013

${ }^{a}$ Department of Medicine, Carmel Medical Center, Faculty of Medicine, Technion, Israel Institute of Technology, Haifa, and Rheumatology Clinic, Nazareth Hospital, Nazareth, Israel

${ }^{\mathrm{b}}$ Department of Medicine, Carmel Medical Center, Haifa, Israel

${ }^{\mathrm{c}}$ Department of Cardiology, Carmel Medical Center, Haifa, Israel

${ }^{\mathrm{d}}$ Corresponding author: George Habib, Department of Medicine,

Carmel Medical Center, Hiafa, 34362, Israel.

Email: gshabib@gmail.com

doi: http://dx.doi.org/10.14740/jmc1336w
}

and coxsackie virus [3]. It was described also following streptococcal infection in few cases [4-8]. The myocardial involvement of this entity adds to the uncertainty about the true diagnosis. Pericarditis "supplies" the chest pain and the ST-segment elevation, while the myocarditis "supplies" the elevated myocardial enzymes and in some cases the regional wall motion abnormality of the myocardium. All these factors might mislead the treating physician towards an incorrect diagnosis of acute coronary event.

Here we report a case of post-streptococcal myopericarditis who was suspected of acute coronary event, and eventually had angiogram of the coronary arteries followed by coronary computerized tomography that showed normal coronary arteries. We will try to highlight important clues in order to help the treating physician to reach a correct diagnosis.

\section{Case Report}

A 45 years old man with hyperlipidemia and family history of coronary artery disease, had throat pain with elevated temperature for 3 days diagnosed with follicular tonsillitis by the family physician. On the third day, patient developed persistent right chest pain exacerbated by deep breathing. At the emergency room electrocardiogram showed elevated ST segment at leads II-III, AVF and V 5-6 with prominent R at V1-2. Chest X-ray was normal. White cell count in the blood was 5,270/mL, GOT $37 \mathrm{U} / \mathrm{L}$, GPT normal, LDH 372 $\mathrm{U} / \mathrm{L}, \mathrm{CPK} 634 \mathrm{U} / \mathrm{L}$ (normal up to 100), troponin $0.46 \mathrm{ng} / \mathrm{mL}$ (normal up to 0.1). C-reactive protein level was $8.61 \mathrm{mg} /$ dL (normal up to 0.5 ). Echocardiogram showed hypokinesia of the infero-lateral wall. Patient was suspected with acute coronary event, treated with anti-aggregants and rushed to the angiography center for coronary angiography that was normal. Patient was started on aspirin $2 \mathrm{~g}$ /day with resolution of his symptoms within $24 \mathrm{~h}$. Repeated echocardiogram 3 days following admission was normal and coronary computerized tomography showed late enhancement of the pericardium at the infero-lateral portion of the left ventricle and posterior portion of the right ventricle. Anti-streptolysin titer was $500.6 \mathrm{IU} / \mathrm{mL}$ decreasing to 161.03 weeks later. Antibodies to echo, adeno and coxsackie viruses were negative 
for acute infection.

\section{Discussion}

Myopericarditis could understandably raise the suspicion of acute coronary events with its electrocardiographic and serum cardiac enzymes. With both differential diagnosis at hand, the treating physician including cardiologists, might be mislead by the echocardiographic findings. Regional wall motion abnormality of the myocardium with the lack of pericardial fluid is usually perceived as more evidence towards coronary event.

Post streptococcal myopericarditis was reported in few cases before [4-8]. All the cases involved were young male patients. In all of them there also was wall motion abnormality of the myocardium, in some regional and in others global. In all of them there also was a full recovery of the myocardial function within days to few weeks. So, regional wall motion abnormality should not deviate the working diagnosis towards coronary event, but should also be considered as part of the presentation of myopericarditis.

Patients with myopericarditis who are suspected for acute coronary event should have immediate cardiac computerized tomography. This modality can rule out with high sensitivity and specificity acute coronary event and can at the same time help with the diagnosis of acute myopericarditis, showing pericardial enhancement, a characteristic finding of pericarditis, and even very small amounts of pericardial effusion, not picked up by echocardiography [9].

\section{References}

1. Mistchenko AS, Maffey AF, Casal G, Kajon AE. Adenoviral pericarditis: high levels of interleukin 6 in pericardial fluid. Pediatr Infect Dis J. 1995;14(11):1007-1009.

2. Shanmugam J, Raveendranath $M$, Balakrishnan KG. Isolation of ECHO virus type-22 from a child with acute myopericarditis--a case report. Indian Heart J. 1986;38(1):79-80.

3. Lee WS, Lee KJ, Kwon JE, Oh MS, Kim JE, Cho EJ, Kim CJ. Acute viral myopericarditis presenting as a transient effusive-constrictive pericarditis caused by coinfection with coxsackieviruses A4 and B3. Korean J Intern Med. 2012;27(2):216-220.

4. Habib G, Cohen L. Myopericarditis associated with streptococcal tonsilitis. Isr Med Assoc J. 2001;3(1):74-75.

5. Said SA, El-Mowafy AM. Role of endogenous endothelin-1 in stress-induced gastric mucosal damage and acid secretion in rats. Regul Pept. 1998;73(1):43-50.

6. Putterman C, Caraco Y, Shalit M. Acute nonrheumatic perimyocarditis complicating streptococcal tonsillitis. Cardiology. 1991;78(2):156-160.

7. Gill MV, Klein NC, Cunha BA. Nonrheumatic poststreptococcal myocarditis. Heart Lung. 1995;24(5):425426.

8. Karjalainen J. Streptococcal tonsillitis and acute nonrheumatic myopericarditis. Chest. 1989;95(2):359-363.

9. Wong BY, Lee KR, MacArthur RI. Diagnosis of pericardial effusion by computed tomography. Chest. 1982;81(2):177-181. 